\title{
Difficult Biliary Cannulation from the Perspective of Post- Endoscopic Retrograde Cholangiopancreatography Pancreatitis: Identifying the Optimal Timing for the Rescue Cannulation Technique
}

\author{
Yoon Suk Lee ${ }^{1}$, Chang Min $\mathrm{Cho}^{2}$, Kwang Bum $\mathrm{Cho}^{3}$, Jun $\mathrm{Heo}^{2}$, Min Kyu Jung², Sung Bum Kim², Kook Hyun Kim², \\ Tae Nyeun Kim${ }^{4}$, Dong Wook Lee ${ }^{5}$, Jimin Han ${ }^{5}$, Ho Gak Kim , Daejin Kim6, and Hyunsoo Kim ${ }^{6}$ \\ ${ }^{1}$ Department of Internal Medicine, Inje University College of Medicine, Goyang, ${ }^{2}$ Department of Internal Medicine, School of Medicine, \\ Kyungpook National University, ${ }^{3}$ Department of Internal Medicine, Keimyung University School of Medicine, ${ }^{4}$ Department of Internal \\ Medicine, Yeungnam University College of Medicine, ${ }^{5}$ Department of Internal Medicine, Daegu Catholic University School of Medicine, \\ and ${ }^{6}$ Department of Internal Medicine, Daegu Fatima Hospital, Daegu, Korea
}

\section{Article Info}

Received September 10, 2019

Revised November 3, 2019

Accepted November 18, 2019

Published online February 3, 2020

\section{Corresponding Author}

Chang Min Cho

ORCID https://orcid.org/0000-0002-9903-1282

E-mail cmcho@knu.ac.kr

The abstract of this study was presented as a poster at 24th United European Gastroenterology Week 2016, on October 15-19, 2016, in Vienna, Austria.
Background/Aims: Recently, the European Society of Gastrointestinal Endoscopy (ESGE) proposed criteria for "difficult biliary cannulation" during endoscopic retrograde cholangiopancreatography (ERCP). This study aimed to investigate the clinical relevance of the ESGE criteria from the perspective of post-ERCP pancreatitis (PEP).

Methods: An ERCP database was prospectively maintained between November 2014 and December 2015 across six teaching hospitals in South Korea. The ESGE criteria (biliary cannulation time, the number of cannulation attempts, and inadvertent pancreatic duct [PD] manipulation) were recorded in this database as well as other technical factors. Logistic regression analysis was used to identify risk factors for PEP. Then, the PEP prediction model was investigated using decision tree analysis.

Results: We analyzed 1,067 consecutive patients with naïve papilla. The overall rate of PEP was $6.6 \%$. Multivariate analysis revealed that female sex (odds ratio [OR], 1.860; 95\% confidence interval [Cl], 1.124 to 3.078), a selective biliary cannulation duration $>5$ minutes (OR, 3.282; $95 \%$ $\mathrm{Cl}, 1.641$ to 6.566 ), and inadvertent PD manipulation (OR, 2.614; $95 \% \mathrm{Cl}, 1.480$ to 4.617 ) were significant factors affecting PEP. Decision tree analysis revealed that biliary cannulation time $\left(\chi^{2}=49.857, p<0.001\right)$ and inadvertent PD manipulation $\left(\chi^{2}=8.556, p=0.010\right)$ were decisive factors. PEP occurred in $3.9 \%, 11.8 \%$, and $16.2 \%$ of patients with biliary cannulation duration lasting 3 to 5 minutes, $>5$ minutes, and $>5$ minutes with inadvertent PD manipulation, respectively.

Conclusions: Biliary cannulation time and inadvertent PD manipulation could be relevant indicators of PEP, and 5 minutes might be used as a cutoff value for the implementation of the rescue cannulation technique. (Gut Liver 2021;15:459-465)

Key Words: Pancreatitis; Cholangiopancreatography, endoscopic retrograde; Risk factors; Decision trees; Practice guideline

\section{INTRODUCTION}

Endoscopic retrograde cholangiopancreatography (ERCP) is a technically challenging procedure for several pancreatobiliary disorders. Reportedly, selective biliary cannulation, which is a prerequisite for successful ERCP, was difficult in $5 \%$ of cases even when performed by expe- rienced endoscopists, and this rate rose to approximately $18 \%$ of cases when performed across low-volume centers. ${ }^{1}$

Difficult biliary cannulation associated with repeated and prolonged attempts increases the risks of ERCP-related adverse events, particularly the risk of post-ERCP pancreatitis (PEP). ${ }^{2,3}$ Recently, the European Society of Gastrointestinal Endoscopy (ESGE) defined "difficult biliary can- 
nulation" as any of the following factors: (1) more than five contacts with the papilla prior to successful cannulation; (2) more than 5 minutes of cannulation time following visualization of the papilla; and (3) more than one inadvertent pancreatic duct (PD) cannulation or opacification. ${ }^{4,5}$ However, the definition of "difficult biliary cannulation" varies across studies and thus interstudy comparisons are impractical. Therefore, the aim of this study was to investigate the clinical relevance of the ESGE's criteria for difficult cannulation from the perspective of post-ERCP pancreatitis.

\section{MATERIALS AND METHODS}

ERCP database from six endoscopy centers in Daegu, South Korea (Kyungpook National University Chilgok Hospital, Kyungpook National University Hospital, Keimyung University Dongsan Medical Center, Yeungnam University Hospital, Daegu Catholic University Hospital, and Daegu Fatima Hospital) was prospectively maintained between November 2014 and December 2015. This database has been registered on the Clinical Research Information Service as clinical research (cris.nih.go.kr number, KCT0003606). All ERCP procedures performed with the intention of accessing the common bile duct to cannulate the naïve papilla were included in this study. However, patients with any of the following criteria were excluded from the study: pregnant women, age $<18$ years, underlying chronic pancreatitis, main PD dilatation measuring $>5$ $\mathrm{mm}$, uncontrolled coagulopathy (international normalized ratio $>1.5$ or platelet count $\left.<50,000 / \mathrm{mm}^{3}\right)$, or medically unstable cardiopulmonary disability for conscious sedation.

The study protocol conforms to the ethical guidelines of the 1975 Declaration of Helsinki as reflected in a priori approval by the Medicity Daegu Joint Institutional Review Board (IRB No. 2018-12-008). All authors had access to the study data and reviewed and approved the final manuscript.

\section{Endoscopic procedure}

ERCPs were performed at each participating hospital by experienced endoscopists who had performed $>200$ therapeutic ERCPs per year. Cannulation techniques (conventional catheter-first with subsequent sphincterotome or sphincterotome-first with/without a preloaded guidewire) and the types of devices used for therapeutic procedures (balloon or basket for stone removal, plastic or selfexpandable metal stent [covered or uncovered] for biliary drainage) were left to the endoscopist's discretion. Moreover, peri-procedural management such as the amount of hydration or type of fluid and prophylactic drug was also left to the strategy of participant hospitals. Endoscopistdirected-nurse administered sedation with midazolam, fentanyl, and/or propofol was used at all participating hospitals.

\section{Procedural outcomes and definitions}

The ERCP database was maintained using a standardized reporting form at all six participating hospitals. Indications, the cannulation technique, therapeutic interventions, results, and adverse events related to the procedure were recorded in all cases. The following data were recorded upon completion of each ERCP: total procedure time, biliary cannulation time, cannulation attempts, contact with papilla, inadvertent PD catheter insertion, PD contrast injection, and PD guidewire passage. Procedurerelated adverse events such as PEP, perforation, bleeding, or others were also recorded based on the definitions proposed by Cotton et al., ${ }^{6,7}$ if these occurred within the index hospitalization period. PEP was defined as the occurrence of new-onset abdominal pain that was associated with an increase in serum amylase at least three times the upper limit of normal at the next day after the procedure. ${ }^{6}$ ERCP-related bleeding was categorized into three subtypes: intraprocedural, immediate, and delayed bleeding. Intraprocedural bleeding was defined when it occurred during the procedure and additional manipulation was required for hemostasis. Immediate bleeding was defined when a hemoglobin drop of at least $2 \mathrm{~g} / \mathrm{dL}$ was observed within 24 hours after the procedure and delayed bleeding was defined when it occurred after 24 hours later. Biliary cannulation time was defined as the duration between the first visualization of the papilla and deep cannulation. Cannulation time was recorded using a built-in stopwatch in the endoscopy system. Cannulation contact was defined as each continuous contact with the papilla with the intention of deep biliary cannulation. Repositioning following a loss of contact with the papilla was defined as a fresh attempt, and the number of cannulation attempts was recorded. In case of inadvertent PD catheter and contrast agent or guidewire passage into the PD occurred, the number of these events was also recorded. Furthermore, inadvertent PD catheter insertion, PD contrast injection, and $\mathrm{PD}$ guidewire passage were categorized as inadvertent PD manipulation because 2016 ESGE guidelines do not distinguish between these criteria in detail.

\section{Statistical analysis}

The independent t-test or the Mann-Whitney U-test was used for continuous variables, and the chi-square or the Fisher exact test was used for categorical variables. Uni- 
variate and multivariate analyses with logistic regression were performed to evaluate patient- and procedure-related variables to determine the independent risk factors associated with PEP. All variables showing a p-value $<0.05$ in the univariate analysis were subjected to multivariate analysis. The results were expressed as odds ratios with $95 \%$ confidence intervals. All p-values were two-sided, and a p-value $<0.05$ was considered statistically significant. All statistical analyses were performed using the SPSS statistics software,

Table 1. Baseline Characteristics of All Enrolled Patients

\begin{tabular}{|c|c|}
\hline Characteristic & Value $(n=1,067)$ \\
\hline Age, yr & $70(19-101)$ \\
\hline Female sex & $451(42.3)$ \\
\hline \multicolumn{2}{|l|}{ Indication for ERCP } \\
\hline Choledocholithiasis & 790 (73.0) \\
\hline Malignant biliary stricture & $238(22.1)$ \\
\hline Benign biliary stricture & 20 (1.9) \\
\hline Post-cholecystectomy bile leakage & $11(1.0)$ \\
\hline Others & $10(0.9)$ \\
\hline Presence of periampullary diverticulum & $315(29.5)$ \\
\hline Type 1 & $46(14.6)$ \\
\hline Type 2 & $142(45.1)$ \\
\hline Type 3 & $127(40.3)$ \\
\hline \multicolumn{2}{|l|}{ Procedure time, $\min$} \\
\hline Overall & $16(3-85)$ \\
\hline Biliary cannulation & $5(1-65)$ \\
\hline \multicolumn{2}{|l|}{ Bile duct access } \\
\hline Successful first attempt & $761(70.4)$ \\
\hline Failed biliary cannulation & $24(2.2)$ \\
\hline \multicolumn{2}{|l|}{ Contacts with papilla } \\
\hline None & $597(56.0)$ \\
\hline$<5$ times & $357(33.5)$ \\
\hline $5-10$ times & 78 (7.3) \\
\hline$\geq 10$ times & $35(3.3)$ \\
\hline \multicolumn{2}{|l|}{ Inadvertent PD catheter insertion } \\
\hline None & $860(80.6)$ \\
\hline$<5$ times & $169(15.8)$ \\
\hline $5-10$ times & $24(2.2)$ \\
\hline$\geq 10$ times & $14(1.3)$ \\
\hline \multicolumn{2}{|l|}{ Inadvertent PD contrast injection } \\
\hline None & 909 (85.2) \\
\hline$<5$ times & $149(14.0)$ \\
\hline 5-10 times & $9(0.8)$ \\
\hline$\geq 10$ times & 0 \\
\hline \multicolumn{2}{|l|}{ Inadvertent PD guidewire passage } \\
\hline None & 767 (71.9) \\
\hline$<5$ times & 258 (24.2) \\
\hline $5-10$ times & $30(2.8)$ \\
\hline$\geq 10$ times & $12(1.1)$ \\
\hline Precut sphincterotomy & $60(5.6)$ \\
\hline Infundibulotomy & $81(7.6)$ \\
\hline Endoscopic papillary balloon dilation & $131(12.3)$ \\
\hline Normal sphincterotomy & 846 (79.3) \\
\hline Prophylactic drugs & $784(73.5)$ \\
\hline Nafamostat mesylate & 362 (33.9) \\
\hline Ulinastin & $228(21.4)$ \\
\hline Gabexate mesylate & 194 (18.2) \\
\hline
\end{tabular}

version 20.0 (IBM Corp., Armonk, NY, USA).

Furthermore, decision tree analysis with the Chisquared Automatic Interaction Detector algorithm was conducted for the hierarchical evaluation of the risk factors of PEP including the criteria proposed by ESGE. In addition to previously known risk factors for PEP including female sex, a history of precut sphincterotomy, and PD contrast injection, other factors that were observed to be significant on univariate analysis were included in the decision tree analysis.

\section{RESULTS}

\section{Baseline characteristics and technical outcomes}

Table 1 summarizes the baseline, clinical, and procedural characteristics (clinical and technical aspects) of the 1,067 consecutive patients with naïve papilla who were included in this study. Common bile duct stones (73.0\%) were the most common indication for ERCP in our study. The second was malignant biliary stricture (238 patients, $22.1 \%$ ), including cholangiocarcinoma (157 patients), pancreatic cancer (38 patients), gallbladder cancer (25 patients), ampulla of Vater cancer (14 patients), and bile duct metastasis (four patients). Selective bile duct cannulation at the first attempt succeeded in 761 patients (70.4\%). The overall success rate for biliary cannulation was $97.4 \%$ (1,040 patients; median time, 5 minutes; range, 1 to 65 minutes). A periampullary diverticulum was identified in $29.5 \%$ of the patients. Precut sphincterotomy (5.6\%) or infundibulotomy $(7.6 \%)$ was performed as a rescue technique for selective bile duct cannulation. Procedure-related adverse events including PEP, post-ERCP bleeding, perforation, and cholangitis occurred in 70 (6.6\%), 120 (11.2\%), seven $(0.6 \%)$, and $14(1.3 \%)$ patients, respectively. The grade of

Table 1. Continued

\begin{tabular}{lc}
\hline \multicolumn{1}{c}{ Characteristic } & Value $(n=1,067)$ \\
\hline Post-ERCP adverse events & $190(17.8)$ \\
Pancreatitis & $70(6.6)$ \\
Mild & $61(87.1)$ \\
Moderate & $7(10.0)$ \\
Severe & $2(2.9)$ \\
Bleeding & $120(11.2)$ \\
Intraprocedural & $114(95.0)$ \\
Immediate & $3(2.5)$ \\
Delayed & $3(2.5)$ \\
Perforation & $7(0.6)$ \\
Cholangitis & $14(1.3)$
\end{tabular}

Data are presented as median (range) or number (\%).

ERCP, endoscopic retrograde cholangiopancreatography; PD, pancreatic duct. 
PEP was mild in $61(87.1 \%)$, moderate in seven $(10.0 \%)$, and severe in two $(2.9 \%)$. Three subtypes of post-ERCP bleeding was as follows: intraprocedural bleeding was occurred in 114 (95\%), immediate in three (2.5\%), and delayed in three $(2.5 \%)$.

\section{Risk factors for post-ERCP pancreatitis}

According to PEP development, the enrolled patients were categorized into two groups (PEP and non-PEP group). Female sex, overall procedure time ( $>10$ minutes), biliary cannulation time ( $>5$ minutes), the number of contacts with the papilla ( $>5$ times), inadvertent PD manipulation (PD contrast injection, guidewire passage, and/ or cannulation), and PD stent placement were statistically significant on univariate analysis. However, overall procedure time ( $>10$ minutes), the number of contacts with the papilla ( $>5$ times) and PD stent placement turned out to be insignificant on multivariate analysis (Table 2).

\section{Decision tree analysis for PEP prediction model}

Decision tree analysis showed that the biliary cannulation time $\left(\chi^{2}=49.857, \mathrm{p}<0.001\right)$ and inadvertent PD manipulation $\left(\chi^{2}=8.556, \mathrm{p}=0.010\right)$ were significantly associated with PEP. However, the number of contacts with the papilla was not significant for discrimination. Moreover, the final decision tree model showed that the biliary cannulation time was the most significant factor (Fig. 1).

We identified that 3 and 5 minutes of biliary cannulation time were the cutoff values for the development of
PEP (3.9\% vs 11.8\%, respectively). Furthermore, if inadvertent $\mathrm{PD}$ manipulation occurred in a patient with biliary cannulation time of $>5$ minutes, the risk of PEP increased to $16.2 \%$ (Fig. 1). However, for the subgroup of moderateto-severe grade PEP, inadvertent PD manipulation was the only significant factor with a $1.9 \%$ risk of moderate-tosevere PEP in the decision tree model (Fig. 2).

\section{DISCUSSION}

This study revealed that the biliary cannulation time and inadvertent PD manipulation were significant factors for PEP development among the 3 ESGE's criteria for difficult biliary cannulation (more than 5 minutes of cannulation time, more than five contacts with the papilla, and/ or more than one inadvertent PD cannulation/opacification). ${ }^{4,5}$ Furthermore, decision tree analysis showed that biliary cannulation time is the most relevant parameter of cannulation difficulty from the perspective of PEP development. PEP occurred in $11.8 \%$ of all cases when the biliary cannulation time was $>5$ minutes. Moreover, the risk of PEP increased to $16.3 \%$ in patients in whom inadvertent PD manipulation occurred when the biliary cannulation time was $>5$ minutes. However, the number of contacts with the papilla was not a significant factor for PEP in multivariate analysis and decision tree analysis. This result may be explained by the fact that the number of contacts with the papilla plays the role of a covariate associated

Table 2. Univariate and Multivariate Analyses of Risk Factors for Post-ERCP Pancreatitis ( $n=1,067)$

\begin{tabular}{|c|c|c|c|c|c|c|}
\hline \multirow[b]{2}{*}{ Variable } & \multicolumn{3}{|c|}{ Univariate analysis } & \multicolumn{3}{|c|}{ Multivariate analysis } \\
\hline & $\begin{array}{l}\text { With PEP } \\
\qquad(n=70)\end{array}$ & $\begin{array}{l}\text { Without PEP } \\
\quad(n=997)\end{array}$ & $\mathrm{p}$-value & Odds ratio & $95 \% \mathrm{Cl}$ & p-value \\
\hline Age $(\leq 70 \mathrm{yr})$ & 39 (55.7) & $499(50.1)$ & 0.364 & & & \\
\hline Female sex & 39 (55.7) & $412(41.3)$ & 0.018 & 1.860 & $1.124-3.078$ & 0.016 \\
\hline Periampullary diverticulum & 18 (25.7) & $297(29.8)$ & 0.470 & & & \\
\hline Failed $B D$ access & $3(4.3)$ & $21(2.1)$ & 0.220 & & & \\
\hline Overall procedure time (>10 $\mathrm{min}$ ) & 66 (94.3) & 755 (75.7) & $<0.001$ & 1.916 & $0.615-5.973$ & 0.262 \\
\hline BD cannulation (>5 min) & $56(80.0)$ & 418 (41.9) & $<0.001$ & 3.282 & $1.641-6.566$ & 0.001 \\
\hline Contact with papilla (>5 times) & $14(20.0)$ & $99(9.9)$ & 0.008 & 0.862 & $0.441-1.684$ & 0.664 \\
\hline Inadvertent PD manipulation & 46 (65.7) & 322 (32.3) & $<0.001$ & 2.614 & $1.480-4.617$ & 0.001 \\
\hline PD catheter insertion ( $\geq 1$ attempt) & $26(37.1)$ & $181(18.2)$ & $<0.001$ & & & \\
\hline PD contrast injection ( $\geq 1$ event) & 19 (27.1) & 139 (13.9) & 0.003 & & & \\
\hline PD guidewire passage ( $\geq 1$ event) & $40(57.1)$ & $260(26.1)$ & $<0.001$ & & & \\
\hline Precut sphincterotomy & $5(7.1)$ & 55 (5.5) & 0.568 & & & \\
\hline EPBD & $4(5.7)$ & $127(12.7)$ & 0.083 & & & \\
\hline Mechanical lithotripsy & $4(5.7)$ & 63 (6.3) & 0.840 & & & \\
\hline ERPD & $9(13.0)$ & $54(5.4)$ & 0.010 & 0.963 & $0.430-2.153$ & 0.926 \\
\hline Prophylactic drug & 59 (84.3) & 725 (72.7) & 0.034 & 1.463 & $0.735-2.912$ & 0.279 \\
\hline
\end{tabular}

Data are presented as number (\%).

ERCP, endoscopic retrograde cholangiopancreatography; PEP, post-ERCP pancreatitis; $\mathrm{Cl}$, confidence interval; $\mathrm{BD}$, bile duct; $\mathrm{PD}$, pancreatic duct; EPBD, endoscopic papillary balloon dilation; ERPD, endoscopic retrograde pancreatic drainage. 


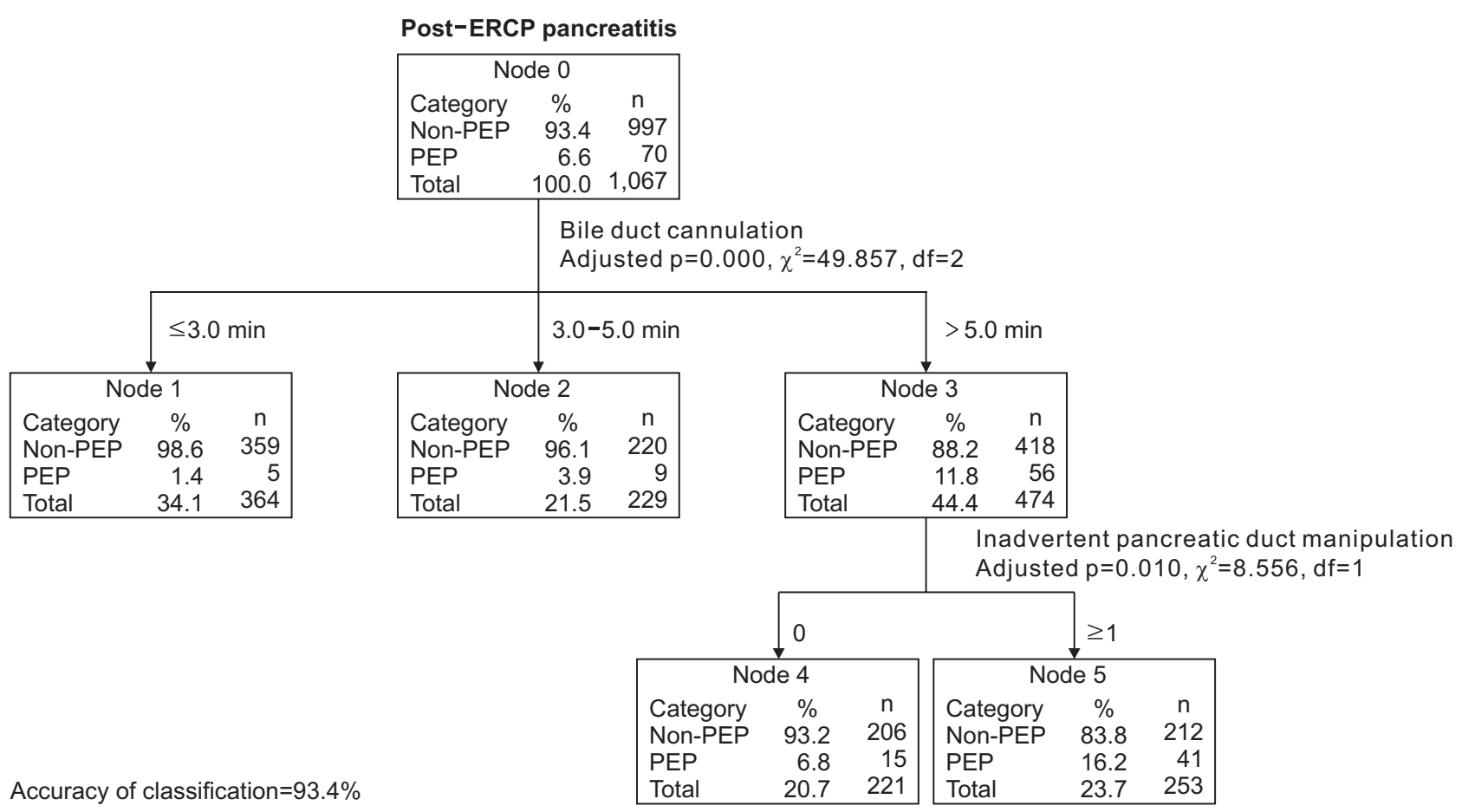

Fig. 1. A decision tree model for the prediction of post-ERCP pancreatitis generated by classification and regression tree analysis using our ERCP database.

ERCP, endoscopic retrograde cholangiopancreatography; PEP, post-ERCP pancreatitis.

Post-ERCP pancreatitis (moderate to severe)

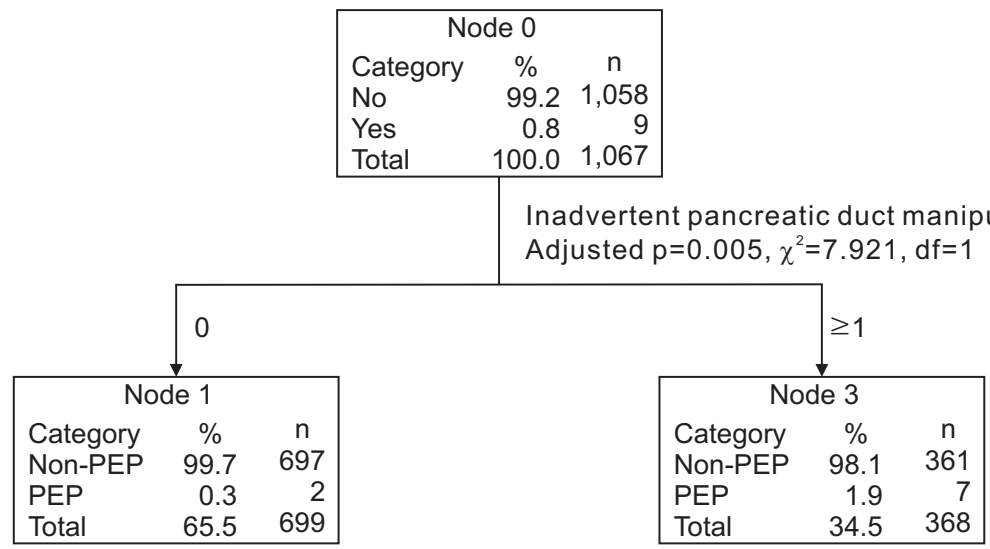

Accuracy of classification $=99.2 \%$
Fig. 2. A decision tree model for the prediction of post-ERCP pancreatitis in the moderate-to-severe grade subgroup, generated by classification and regression tree analysis using our ERCP database.

ERCP, endoscopic retrograde cholangiopancreatography; PEP, postERCP pancreatitis. with the biliary cannulation time. This finding is also supported by the study of Tian et al. ${ }^{8}$ which demonstrated that there was a positive correlation between cannulation time and the number of attempts, and cannulation time was a more objective and accurate assessment tool for cannulation difficulty. Furthermore, the low number of contacts with papilla does not necessarily mean easy cannulation. Therefore, among the 3 ESGE's criteria for difficult cannulation proposed in 2016, the biliary cannulation time and inadvertent PD manipulation would be indicators of PEP development; and then 5 minutes could be used as a cutoff value for the implementation of rescue cannulation technique.

Recently, Mariani et al. ${ }^{9}$ reported that early precut sphincterotomy (in which rescue needle-knife sphincterotomy was performed whenever bile duct cannulation failed after 5 minutes or after three passes of inadvertent PD cannulation), reduced the incidence of PEP. This finding suggests that precut sphincterotomy by itself is not a risk factor, but prolonged cannulation might be a more significant 
risk factor for PEP. These findings were in agreement with our study outcomes for reducing the cannulation time to prevent PEP. When decision tree analysis was performed only for moderate-to-severe PEP, inadvertent PD manipulation was the only factor that showed statistical significance, which might indicate that instrumentation and manipulation-induced mechanical injury to the PD is the most important pathophysiological contributor to moderate-to-severe PEP rather than electrosurgery-induced thermal injury associated with sphincterotomy. Furthermore, two recent meta-analyses of randomized controlled trials have also demonstrated the benefit of early precut sphincterotomy to prevent PEP. ${ }^{10,11}$ Therefore, endoscopists should try to reduce the time of selective biliary cannulation as short as possible and, if not, rescue techniques such as precut sphincterotomy or double guidewire technique should be considered in an early phase of the procedure in the case of difficult biliary cannulation.

Considering the association between inadvertent PD manipulation and PEP, which was also demonstrated in this study, the insertion of a prophylactic indwelling PD stent is a reasonable recommendation. However, pancreatic stent and drugs for PEP prophylaxis were ineffective in this study. This finding might be explained by the observational study design in that certain patients with a seemingly high probability for PEP might be more frequently treated with prophylactic medications or PD stent based on personal criteria.

The limitations of this study are as follows: (1) this study was designed as an observational study although the data were collected prospectively. However, we used a relatively large multicenter ERCP database containing detailed information and technical data regarding procedures performed by highly qualified and experienced endoscopists. (2) The method of and devices used for biliary cannulation as well as the strategy of peri-procedural management such as the amount of hydration or type of fluid and prophylactic drug were not standardized across all participating hospitals and operators. However, all endoscopists who performed the procedures included in this study were highly experienced, which was reflected by the ERCP-related technical outcomes of this study (the success rate of biliary cannulation was $97.5 \%$, and the median biliary cannulation time was 5 minutes). Therefore, the variability in cannulation techniques and devices might not have significantly affected our study results.

Nonetheless, the strength of our study is that multicenter ERCP registry from high-volume endoscopy centers was used for the evaluation of difficult cannulation criteria. Thus, the results accurately reflect in detail the technical aspects of the procedure performed by experienced endoscopists. Furthermore, the use of decision tree analysis to identify the risk factors for PEP provided a clear understanding of the hierarchy of the related variables. ${ }^{12-14}$ Decision tree analysis has been frequently used in many medical types of research because it provides an optimal prediction model with identifying relevant factors for primary outcomes. Furthermore, the first discriminating variable of final decision tree model is considered to have the strongest statistical association with the primary outcome variable. ${ }^{15,16}$

In conclusion, among the 3 ESGE's criteria for difficult cannulation, biliary cannulation time and inadvertent PD manipulation would be indicators of PEP development. Furthermore, the biliary cannulation time was the most significant factor for PEP. Therefore, the cannulation time should be as short as possible for the prevention of PEP development; and then 5 minutes might be used as a cutoff value for the implementation of rescue cannulation technique.

\section{CONFLICTS OF INTEREST}

C.M.C. is an editorial board member of the journal but did not involve in the peer reviewer selection, evaluation, or decision process of this article. No other potential conflicts of interest relevant to this article were reported.

\section{ACKNOWLEDGEMENTS}

This work was supported by the National Research Foundation of Korea (NRF) grant funded by the Korea government (MSIP; Ministry of Science, ICT \& Future Planning) (number: 2017R1C1B5018180).

\section{AUTHOR CONTRIBUTIONS}

Study conception and design: Y.S.L., C.M.C. Data collection: Y.S.L., C.M.C., K.B.C., J.H., M.K.J., S.B.K., K.H.K., D.W.L., J.H., D.K., H.K. Analysis and interpretation of the data: Y.S.L., C.M.C. Drafting of the article: Y.S.L., C.M.C. Critical revision of the article for important intellectual content: C.M.C., K.B.C., D.K., H.K., T.N.K., H.G.K. Final approval of the article: all authors.

\section{ORCID}

Yoon Suk Lee https://orcid.org/0000-0002-5835-9417 Chang Min Cho https://orcid.org/0000-0002-9903-1282 
Kwang Bum Cho https://orcid.org/0000-0003-2203-102X Jun Heo https://orcid.org/0000-0003-3180-1655 Min Kyu Jung https://orcid.org/0000-0001-8749-408X Sung Bum Kim https://orcid.org/0000-0001-8447-2176 Kook Hyun Kim https://orcid.org/0000-0001-7786-7882 Tae Nyeun Kim https://orcid.org/0000-0003-4178-2056 Dong Wook Lee https://orcid.org/0000-0002-1029-9064 Jimin Han https://orcid.org/0000-0001-8674-370X Ho Gak Kim https://orcid.org/0000-0003-3365-1662 Daejin Kim https://orcid.org/0000-0001-7554-1870 Hyunsoo Kim https://orcid.org/0000-0001-5572-5453

\section{REFERENCES}

1. Williams EJ, Taylor S, Fairclough P, et al. Are we meeting the standards set for endoscopy? Results of a large-scale prospective survey of endoscopic retrograde cholangio-pancreatograph practice. Gut 2007;56:821-829.

2. Halttunen J, Meisner S, Aabakken L, et al. Difficult cannulation as defined by a prospective study of the Scandinavian Association for Digestive Endoscopy (SADE) in 907 ERCPs. Scand J Gastroenterol 2014;49:752-758.

3. Freeman ML, DiSario JA, Nelson DB, et al. Risk factors for post-ERCP pancreatitis: a prospective, multicenter study. Gastrointest Endosc 2001;54:425-434.

4. Dumonceau JM, Andriulli A, Elmunzer BJ, et al. Prophylaxis of post-ERCP pancreatitis: European Society of Gastrointestinal Endoscopy (ESGE) guideline: updated June 2014. Endoscopy 2014;46:799-815.

5. Testoni PA, Mariani A, Aabakken L, et al. Papillary cannulation and sphincterotomy techniques at ERCP: European Society of Gastrointestinal Endoscopy (ESGE) clinical guideline. Endoscopy 2016;48:657-683.

6. Cotton PB, Lehman G, Vennes J, et al. Endoscopic sphincterotomy complications and their management: an attempt at consensus. Gastrointest Endosc 1991;37:383-393.

7. Cotton PB, Garrow DA, Gallagher J, Romagnuolo J. Risk factors for complications after ERCP: a multivariate analysis of 11,497 procedures over 12 years. Gastrointest Endosc
2009;70:80-88

8. Tian C, Gamboa A, Chaudhury B, Willingham FF, Keilin S, Cai Q. Cannulation time is a more accurate measure of cannulation difficulty in endoscopic retrograde cholangiopancreatography than the number of attempts. Gastroenterol Rep (Oxf) 2013;1:193-197.

9. Mariani A, Di Leo M, Giardullo N, et al. Early precut sphincterotomy for difficult biliary access to reduce post-ERCP pancreatitis: a randomized trial. Endoscopy 2016;48:530535.

10. Sundaralingam P, Masson P, Bourke MJ. Early precut sphincterotomy does not increase risk during endoscopic retrograde cholangiopancreatography in patients with difficult biliary access: a meta-analysis of randomized controlled trials. Clin Gastroenterol Hepatol 2015;13:1722-1729.

11. Tang Z, Yang Y, Yang Z, Meng W, Li X. Early precut sphincterotomy does not increase the risk of adverse events for patients with difficult biliary access: a systematic review of randomized clinical trials with meta-analysis and trial sequential analysis. Medicine (Baltimore) 2018;97:e12213.

12. Hess KR, Abbruzzese MC, Lenzi R, Raber MN, Abbruzzese JL. Classification and regression tree analysis of 1000 consecutive patients with unknown primary carcinoma. Clin Cancer Res 1999;5:3403-3410.

13. Garzotto M, Beer TM, Hudson RG, et al. Improved detection of prostate cancer using classification and regression tree analysis. J Clin Oncol 2005;23:4322-4329.

14. Austin PC. A comparison of regression trees, logistic regression, generalized additive models, and multivariate adaptive regression splines for predicting AMI mortality. Stat Med 2007;26:2937-2957.

15. Rokach L, Maimon O. Data mining with decision trees: theory and applications. Hackensack: World Scientific Publishing, 2014.

16. De Melo NB, Bernardino ÍM, de Melo DP, Gomes DQC, Bento PM. Head and neck cancer, quality of life, and determinant factors: a novel approach using decision tree analysis. Oral Surg Oral Med Oral Pathol Oral Radiol 2018;126:486493. 